\title{
Jet rotation: Launching region, angular momentum balance and magnetic properties in the bipolar outflow from RW Aur ${ }^{\star}$
}

\author{
J. Woitas ${ }^{1}$, F. Bacciotti ${ }^{2}$, T. P. Ray ${ }^{3}$, A. Marconi ${ }^{2}$, D. Coffey ${ }^{3}$, and J. Eislöffel ${ }^{1}$ \\ 1 Thüringer Landessternwarte Tautenburg, Sternwarte 5, 07778 Tautenburg, Germany \\ e-mail: woitas@tls-tautenburg.de \\ 2 INAF - Osservatorio Astrofisico di Arcetri, Largo E. Fermi 5, 50125 Firenze, Italy \\ 3 School of Cosmic Physics, Dublin Institute for Advanced Studies, 5 Merrion Square, Dublin 2, Ireland
}

Received 3 October 2003 / Accepted 28 October 2004

\begin{abstract}
Using STIS on board the HST we have obtained a spectroscopic map of the bipolar jet from RW Aur with the slit parallel to the jet axis and moved across the jet in steps of $0{ }^{\prime} 07$. After applying a velocity correction due to uneven slit illumination we find signatures of rotation within the first $300 \mathrm{AU}$ of the jet (1".5 at the distance of RW Aur). Both lobes rotate in the same direction (i.e. with different helicities), with toroidal velocities in the range $5-30 \mathrm{~km} \mathrm{~s}^{-1}$ at 20 and 30 AU from the symmetry axis in the blueshifted and redshifted lobes, respectively. The sense of rotation is anti-clockwise looking from the tip of the blue lobe (PA $130^{\circ}$ north to east) down to the star. Rotation is more evident in the [OI] and [NII] lines and at the largest sampled distance from the axis. These results are consistent with other STIS observations carried out with the slit perpendicular to the jet axis, and with theoretical simulations.

Using current magneto-hydrodynamic models for the launch of the jets, we find that the mass ejected in the observed part of the outflow is accelerated from a region in the disk within about $0.5 \mathrm{AU}$ from the star for the blue lobe, and within 1.6 AU from the star for the red lobe. Using also previous results we estimate upper and lower limits for the angular momentum transport rate of the jet. We find that this can be a large fraction (two thirds or more) of the estimated rate transported through the relevant portion of the disk. The magnetic lever arm (defined as the ratio $r_{\mathrm{A}} / r_{0}$ between the Alfvèn and footpoint radii) is in the range 3.5-4.6 (with an accuracy of 20-25\%), or, alternatively, the ejection index $\xi=\mathrm{d} \ln \left(\dot{M}_{\text {acc }}\right) / \mathrm{d} r$ is in the range $0.025-0.046$ (with similar uncertainties). The derived values are in the range predicted by the models, but they also suggest that some heating must be provided at the base of the flow.

Finally, using the general disk wind theory we derive the ratio $B_{\phi} / B_{\mathrm{p}}$ of the toroidal and poloidal components of the magnetic field at the observed location (i.e. about $80-100 \mathrm{AU}$ above the disk). We find this quantity to be $3.8 \pm 1.1$ at $30 \mathrm{AU}$ from the axis in the red lobe and $-8.9 \pm 2.7$ at $20 \mathrm{AU}$ from the axis in the blue lobe (assuming cylindrical coordinates centred on the star and with positive $z$ along the blue lobe). The toroidal component appears to be dominant, which would be consistent with magnetic collimation of the jet. The field appears to be more tightly wrapped on the blue side.
\end{abstract}

Key words. ISM: Herbig-Haro objects - ISM: jets and outflows - stars: formation - stars: pre-main sequence - stars: individual: RW Aur

\section{Introduction}

The collimated Herbig-Haro (HH) jets observed on parsecscale lengths in star formation regions are always associated with young stellar objects (YSOs) that are still in their accretion phase. Accretion and ejection of matter are believed to be intimately related phenomena, through the presence of a magnetized accretion disk. The complex interplay between accretion and ejection is modelled using several theoretical approaches (cf. Camenzind et al. 1990; Ferreira 1997; Königl \& Pudritz 2000; Shu et al. 2000, and references therein). These

* Based on observations made with the NASA/ESA Hubble Space Telescope, obtained at the Space Telescope Science Institute, which is operated by the Association of Universities for Research in Astronomy, Inc., under NASA contract NAS5-26555. models have in common the idea that the jet is generated through the interaction of plasma with rotating magnetic field lines that are anchored to the star/disk system. The fluid particles lifted from the disk are forced to slide along the rotating magnetic field lines, and are accelerated and collimated into bipolar jets. The most attractive aspect of this approach is the fact that the magneto-centrifugal scenario at the same time justifies the acceleration of the jets and the extraction of the excess angular momentum from the disk. This mechanism, in combination with disk viscosity (the nature of which, however, is still uncertain), should contribute to slow the disk material down to sub-Keplerian rotation. In this way matter is allowed to move radially toward the central star and finally accrete onto it.

This compelling theoretical picture, however, has received little in the way of observational confirmation, as pointed out 
by Eislöffel et al. (2000). The main reason is that the process occurs at small distances, corresponding, for the nearest star formation regions (130-150 pc), to subarcsecond scales.

The "core" of the central engine lies within a few AU from the source, which is not spatially resolvable with current observational techniques. Important constraints on the launching region, however, can be deduced from the quantities observed in the first 100-200 AU of the flow, in which the jet achieves its collimation and its final poloidal velocity. This region has been accessed recently with high angular resolution observations of jets from evolved T Tauri stars. Such objects have been observed from space with the Hubble Space Telescope Imaging Spectrograph (HST/STIS, 0.'1 resolution, e.g. Bacciotti et al. 2000; Woitas et al. 2002), and from the ground using large telescopes with Adaptive Optics (e.g. Dougados et al. 2000).

Among the properties investigated in the above studies, a special place is taken by the detection and analysis of the rotation of the jets around their symmetry axis (Bacciotti et al. 2002; Testi et al. 2002; Anderson et al. 2003; Coffey et al. 2004; Pesenti et al. 2004; Cerqueira \& de Gouveia Dal Pino 2004). These studies have shown that the measured toroidal velocities are similar to the values predicted by the disk-wind magneto-centrifugal models. The obtained measures of rotation, however, also help to constrain the properties of the accretion/ejection "machine". For example, in combination with mass density estimates from line ratios, rotation profiles can yield the amount of angular momentum carried away from the accreting system by the jet. This can then be compared with the disk accretion properties. As another example, the application of special conservation laws can in principle give information on the properties of the magnetic field in the jet, a quantity that is fundamental to all jet acceleration models, but that is notoriously difficult to directly examine by observation.

The aim of this paper is to illustrate the potential offered by such a combined observational/theoretical investigation to constrain the accretion/ejection structure at the origin of the jet. First, we present results from a new observational study of the RW Aur jet, conducted with multiple slits oriented along the flow axis (Sects. 2 and 3). The adopted technique is similar to the one used in our first rotation study, that concerned the jet from DG Tauri (Bacciotti et al. 2002). Here, however, the technique is applied for the first time to a bipolar jet, on a more extended and more finely sampled region. In addition, more details are given about the correction routines for uneven slit illumination. Then, in the second part of the paper (Sect. 4), we combine the obtained results with morphological and excitation properties of this jet derived on subarcsecond scales from previous studies and partly based on the same dataset (Woitas et al. 2002, hereafter Paper I; Dougados et al. 2000). In this way we show how, using general theoretical principles, one can derive physical quantities that are crucial for a description of the accretion/ejection region close to the origin of the jet. Finally, in Sect. 5 we summarize our conclusions.

\section{Observations and data analysis}

The HST/STIS observations of RW Aur were carried out on 10 December 2000. Details of the data acquisition and

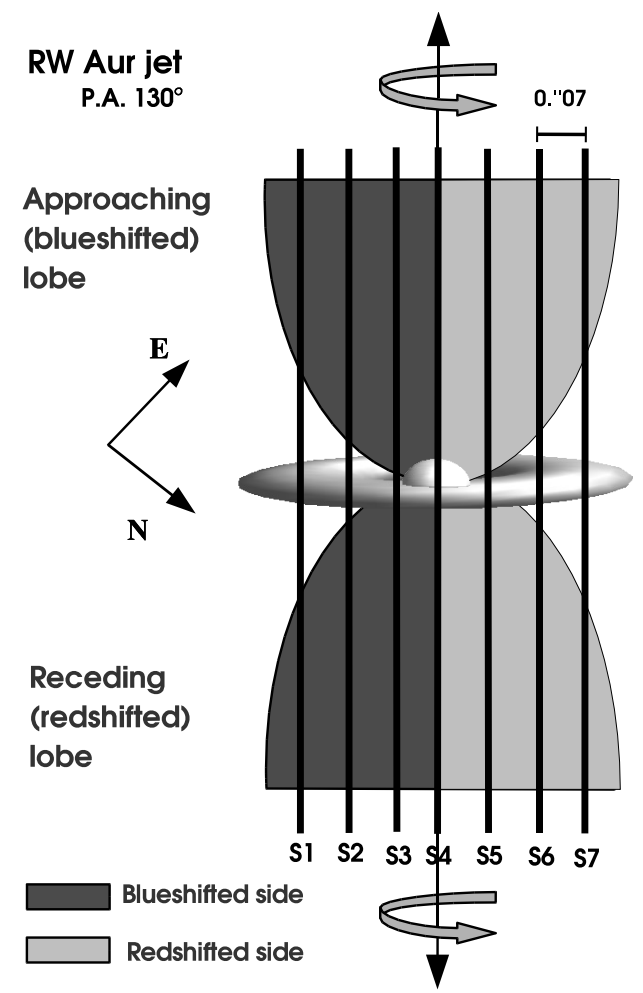

Fig. 1. Schematic drawing of the observing mode for the bi-polar jet from RW Aur. The STIS slit is kept parallel to the flow axis, and stepped sequentially in seven different positions (labelled $S 1, S 2 \ldots S 7$ ) each time by $0{ }^{\prime} 07$. The slit width is $0{ }^{\prime} 1$, so the apertures are slightly overlapping. The sense of rotation derived in the present paper is illustrated by the arrows around the symmetry axis.

reduction were described in Paper I. Briefly, the dataset consists of seven spectra (hereafter $S 1, \ldots, S 7$ ) taken at slit positions parallel to the jet axis $\left(\mathrm{PA}=130^{\circ}\right.$, Dougados et al. 2000). This observing procedure is shown in Fig. 1. The pixel scale is 0. '05/pixel for the spatial axis and $0.554 \AA /$ pixel for the dispersion axis. The latter value corresponds to $\approx 25 \mathrm{~km} \mathrm{~s}^{-1} /$ pixel. The slit width is $0{ }^{\prime} 1$. The covered spectral range contains $\mathrm{H} \alpha$ and the prominent forbidden emission lines [OI] $\lambda \lambda 6300,6363,[\mathrm{NII}] \lambda \lambda 6548,6583$ and [SII] $\lambda \lambda 6716,6731$. However, blueshifted [OI] $\lambda 6300$ emission cannot be used for the present analysis because the line profile is cut by the detector edge.

In the course of the observations the slit is moved transversely across the jet in steps of 0 ' $^{\prime} 07$ from southwest $(S 1)$ to northeast (S7). The position of $S 4$ corresponds to the jet axis. If the jet rotates, one expects non-zero peak velocity differences $S 7-S 1, S 6-S 2$ and $S 5-S 3$ that have the same sign at all separations from the star. However, one has to take into account that uneven slit illumination may introduce spurious velocity shifts causing peak velocity differences similar to those described above, which may then be present also in the case of a non-rotating jet. This effect has been eliminated applying correction routines developed by one of us (A.M.), which also have been used to study the rotational motions in the DG Tau jet. The calculations performed in the correction routine are illustrated in Appendix A of Bacciotti et al. (2002) 
and in Marconi et al. (2003), and the procedure can be briefly summarized in three steps as follows.

(i) In the first step a bidimensional surface $I_{\lambda}(x, y)$ is calculated that simulates the observed brightness distribution in each emission line at the positions $(x, y)$ on the sky intercepted by the seven STIS slits. This is done by fitting separately for each line a series of 2-D surfaces with an elliptical base simulating the knots along the flow to the line fluxes measured along the seven slits at once. The best fit is found through an iterative algorithm. It is then necessary to assume a model radial velocity field of the nebula $u(x, y)$. In our case we wish to determine the spurious velocity caused by STIS, so we assume an arbitrary constant value $\bar{u}$, equal for all the slits and positions on the sky, i.e. a non-rotating uniformly moving jet is assumed.

(ii) In the second step the routines calculate the average velocity measured at the detector $v_{\lambda}(x, y)$ for each slit, line and position considered. This is done by convolving the model velocity field as it would be seen at the detector without optical distortions with the model of the brightness distribution obtained at step (i), with the Point Spread Function of HST at the wavelength of interest and with the slit aperture offset with respect to the axis of the jet as in the observations. Details of this calculation can be found in the Appendix of Bacciotti et al. (2002). Since the model velocity of the gas is set to be the same value for all the slits, the searched instrumental spurious velocity is $V_{\lambda}(x, y)=v_{\lambda}(x, y)-\bar{u}$, the result being independent of the chosen value of the model radial velocity. The absolute values of $V_{\lambda}(x, y)$ range between 2 and $8 \mathrm{~km} \mathrm{~s}^{-1}$ and have opposite sign depending on the relative position of the slit and the jet axis, since slits to the left or to the right of the axis have opposite illumination gradients. The values for $V_{\lambda}(x, y)$ are higher (in absolute value) where the illumination gradient is steeper across the jet, i.e. typically at the position of the knot peaks.

(iii) Finally, the spurious "shift" is calculated between the considered opposed slits as, e.g. $V_{\lambda}(S 7, y)-V_{\lambda}(S 1, y)$. The calculated shifts are instrumental offsets, and therefore they are subtracted from the raw shift measurements, to cancel the effect of the uneven slit illumination.

The spurious velocity depends only on the gradient of the slit illumination, and not on the integrated flux across the slit, so any systematic error in the photometry would not affect our determination. Therefore, the uncertainty associated with the correction depends only on the accuracy with which the shape of the observed emission distribution is fitted by the assumed 2-D surfaces convolved with the instrumental response. The other parameters entering the calculation (see Bacciotti et al. 2002), i.e. the instrument PSF, the detector scale factor and the line flux measured by STIS are all known with a much higher accuracy than that associated with the fitting procedure.

The accuracy of the correction has been estimated a posteriori by investigating the variations in the determined spurious shifts due to an imposed change in the scale factor of the brightness distribution of the surface fitting each knot. Variations of the adopted illumination gradient producing a change by $10-15 \%$ in the value of the fitted flux, which is about the accuracy of our iterative fitting algorithm, lead to a change of $5-8 \%$ in the determination of the spurious velocity shift. Thus we take this factor as the uncertainty of the correction, which, in the case of the largest spurious shifts $\left(\sim 16 \mathrm{~km} \mathrm{~s}^{-1}\right)$, corresponds to less than $\pm 1.5 \mathrm{~km} \mathrm{~s}^{-1}$. Also, we checked that imposed small misplacements of the slit positions or inclination (up to $10 \%$ ) do not lead to any appreciable change in the determination of the spurious velocity.

It should be noted that in the real case in which the velocity field is not constant, an additional deformation is introduced by the passage of the light through the telescope and spectrograph. In our case, however, this contribution can be neglected because we are actually interested in spurious velocity differences between symmetrically opposed slit pairs, and the additional contribution would cancel in the difference because the absolute value of the real radial velocity, as well as the intensity, have a symmetrical variation with respect to the jet axis or central slit.

In practice, the spurious velocity is positive for the slits $S 1$, $S 2$ and $S 3$, located south-west of the axis, and negative for $S 5$, $S 6, S 7$. So for the adopted configuration $(S 7-S 1, S 6-S 2$, $S 5-S 3$ ) the instrument produces a spurious negative shift in both jet lobes. The effect is more pronounced for the forbidden lines (FELs) than it is for $\mathrm{H} \alpha$. This is due to the fact that the bright $\mathrm{H} \alpha$ emission is more evenly distributed across the transverse direction in the area covered by the seven slits (see Paper I). Similarly, other detected differences in the spurious shifts for the different lines can be attributed to the different transverse spatial distribution of the emission (see Table 2 and its description in Sect. 3).

After subtracting the spurious negative shifts the rotation signature becomes more evident for the outermost slit pairs (larger positive shift), while in the innermost pair the measured raw negative shifts are turned back to weakly positive or null (i.e. consistent with zero inside the measurement error of $\left.\pm 7 \mathrm{~km} \mathrm{~s}^{-1}\right)$. In some positions of the innermost pairs, however, the net shift in [SII] and $\mathrm{H} \alpha$ lines remains significantly negative. For an explanation see Sect. 3.

The validity of the illumination correction can be tested using the $\mathrm{H} \alpha$ emission at the stellar position. As this emission is close to saturation, the line profiles will be dominated by the HST PSF and are not supposed to show any rotation signatures. Any velocity shifts between lateral slits will be due to instrumental effects. The finding that there are no significant velocity shifts in $\mathrm{H} \alpha$ close to the star after applying the illumination correction (see Fig. 4) thus strongly indicates that this correction routine really removes velocity shifts caused by uneven slit illumination.

The peak velocities of individual lines in all spectra and in both outflow lobes were determined from Gaussian fits to the line profiles. Many T Tauri jets show two velocity components of forbidden line emission, a high velocity component (HVC) with $v_{r} \approx 100-200 \mathrm{~km} \mathrm{~s}^{-1}$ and an additional low velocity component (LVC) with a typical radial velocity of 5 to $20 \mathrm{~km} \mathrm{~s}^{-1}$ (Hirth et al. 1997). The HVC is thought to come from a collimated jet close to the star, while the LVC might be the signature of a poorly collimated disk wind (Kwan \& Tademaru 1988, 1995). In Paper I we have, however, demonstrated that there is no significant separate LVC in our data. Therefore fitting single Gaussians is sufficient. As an example we show in Fig. 2 the fit to the [SII] $\lambda 6731$ line at slit position $S 3$ and 


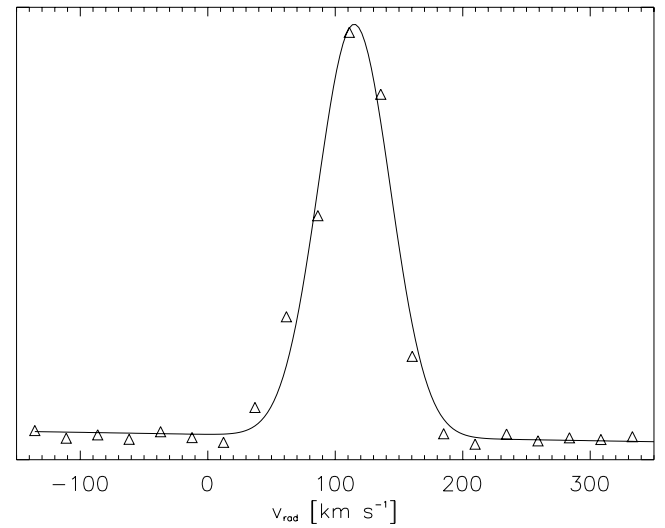

Fig. 2. Example of a Gaussian fit to the line profile that yields the peak velocity. This plot shows [SII] $\lambda 6731$ at slit position S3 and at a separation of $0 . ' 9$ from the star.

separation 0.9 in the redshifted lobe. The uncertainty of the peak velocities is about $\pm 5 \mathrm{~km} \mathrm{~s}^{-1}$, and the uncertainties of velocity differences are thus $\pm 7 \mathrm{~km} \mathrm{~s}^{-1}$.

For some selected positions and emission lines we have derived velocity differences also by means of a cross-correlation technique that directly measures the displacement of two line profiles from different slits, with a typical accuracy of $5 \mathrm{~km} \mathrm{~s}^{-1}$ on the velocity difference. This method is in principle more robust than Gaussian fitting as no special shape of the line profile has to be assumed. Its usefulness is limited, however, in situations where the velocity range is only marginally sampled, and this is the case here with the narrow emission lines of the RW Aur jet. Thus cross-correlation does not lead to a distinct improvement, but the results are consistent with those obtained from the Gaussian fits. Reliable measurements are not possible for all slit positions and separations from the source. The RW Aur jet has a FWHM of about 20 AU within its first arcsecond and is thus more strongly collimated than the jet of DG Tau ( $F W H M \approx 50 \mathrm{AU}$ at $d=0.5$, see Paper I, Fig. 4 therein). For this reason, there are cases where there is no significant signal at distinct separations in $S 1$ and $S 7$. In [NII], which traces emission very close to the jet axis, this will even be the case for more central slit positions. Furthermore, quenching effects make the FELs extremely weak within $0.1-0.2$ from the origin, thus we exclude this region from the analysis. Although the jet is clearly visible up to $\approx 4^{\prime \prime}(\approx 800 \mathrm{AU})$ from the star in the STIS data (Paper I) we restrict the rotation analysis to the first $\approx 300 \mathrm{AU}$ approximately. Over this separation range our data will reflect signatures from the initial jet channel, whereas further away interaction between the jet and the circumstellar environment might become important. To obtain de-projected separations we assume the inclination angle of the RW Aur jet to be $i=46 \pm 3^{\circ}$ with respect to the line of sight (López-Martín et al. 2003). In Paper I we adopted $i=53^{\circ}$, but this estimate was based on the proper motion of only one jet knot and furthermore affected by an erroneous pixel scale given by Dougados et al. (2000) for their 1997 observations (the corrected value is given by López-Martín et al. 2003). With the new value of the inclination angle, 1 '.5 on the sky corresponds to $300 \mathrm{AU}$ along the outflow direction.
Table 1. Mean velocity differences (in $\mathrm{km} \mathrm{s}^{-1}$ ), averaged over all lines and all separations from the star.

\begin{tabular}{llll}
\hline \hline & $S 7-S 1$ & $S 6-S 2$ & $S 5-S 3$ \\
\hline Redshifted lobe & $12.9 \pm 1.7$ & $0.2 \pm 0.8$ & $2.0 \pm 0.6$ \\
Blueshifted lobe & $5.2 \pm 1.3$ & $1.1 \pm 1.6$ & $3.8 \pm 0.9$ \\
\hline
\end{tabular}

\section{Indications of rotation}

Figure 3 shows the peak velocities for all slit positions and at different separations from the star. These velocities are corrected for uneven slit illumination (see Sect. 2) and for the heliocentric radial velocity of RW Aur A, i.e. $+23.5 \mathrm{~km} \mathrm{~s}^{-1}$ as derived from the Li I line at $\lambda \approx 6707 \AA$ from our data. We note that Petrov et al. (2001) repeatedly observed RW Aur using high resolution echelle spectroscopy and found a mean heliocentric $v_{r}$ of $+16 \mathrm{~km} \mathrm{~s}^{-1}$ with periodic variations of $\pm 6 \mathrm{~km} \mathrm{~s}^{-1}$ $(P \approx 2.8 \mathrm{~d})$. Such variation in the adopted systemic velocity does not affect our determinations of velocity differences between the two sides of the flow. For the present analysis, however, in which we also give transverse profiles of the absolute radial velocity, we decided to adopt the former value for selfconsistency.

The resulting velocity differences $S 7-S 1, S 6-S 2$ and $S 5-S 3$ are plotted in Figs. 4 and 5 as a function of the separation from the star. The result is not as impressive as for DG Tau, due to the fact that the RW Aur jet is more collimated (see below). One can see, however, that in all slit pairs there is a definite tendency for the velocity shifts to be located on the same side of the zero line in both lobes. The scatter of the data points in the different lines is sometimes large, and it is probably due to different excitation conditions, as discussed later. Nevertheless, the averages of all velocity shifts in all lines and separations from the star show the same sign in all slit pairs and in both lobes (Table 1). Thus, the emission from the northeastern parts of both lobes is more redshifted than the emission from the south-western parts. This leads us to suggest that both outflow lobes rotate clockwise looking from the tip of the redshifted lobe down to the star. The matter thus flows in the jet with opposite helicities. These results are fully consistent with the study in Coffey et al. (2004).

Given the fact that the measured rotation velocities are of the order of only $\approx 10 \mathrm{~km} \mathrm{~s}^{-1}$ one has to look carefully at artifacts that might mimic rotation. The problem of uneven slit illumination has already been discussed in Sect. 2. Another issue that has to be taken into account here is that the slit orientation might not be exactly parallel to the jet axis. Gaussians fits to the emission profiles transverse to the jet, as well as the output of the illumination correction routines, do indeed show that the slit set is well-centred on the star, but the chosen slit PA (130 deg) turns out to be larger by 1-2 degrees than the real PA of the jet direction (blue lobe - the same misalignment is also noted in Coffey et al. 2004). This causes the slits of one given "pair" to trace gas with different poloidal velocity, which will lead to apparent velocity shifts even if the jet is nonrotating. From Fig. 3 one can estimate that the corresponding "false" shifts would be $1-5 \mathrm{~km} \mathrm{~s}^{-1}$, which is not negligible. 

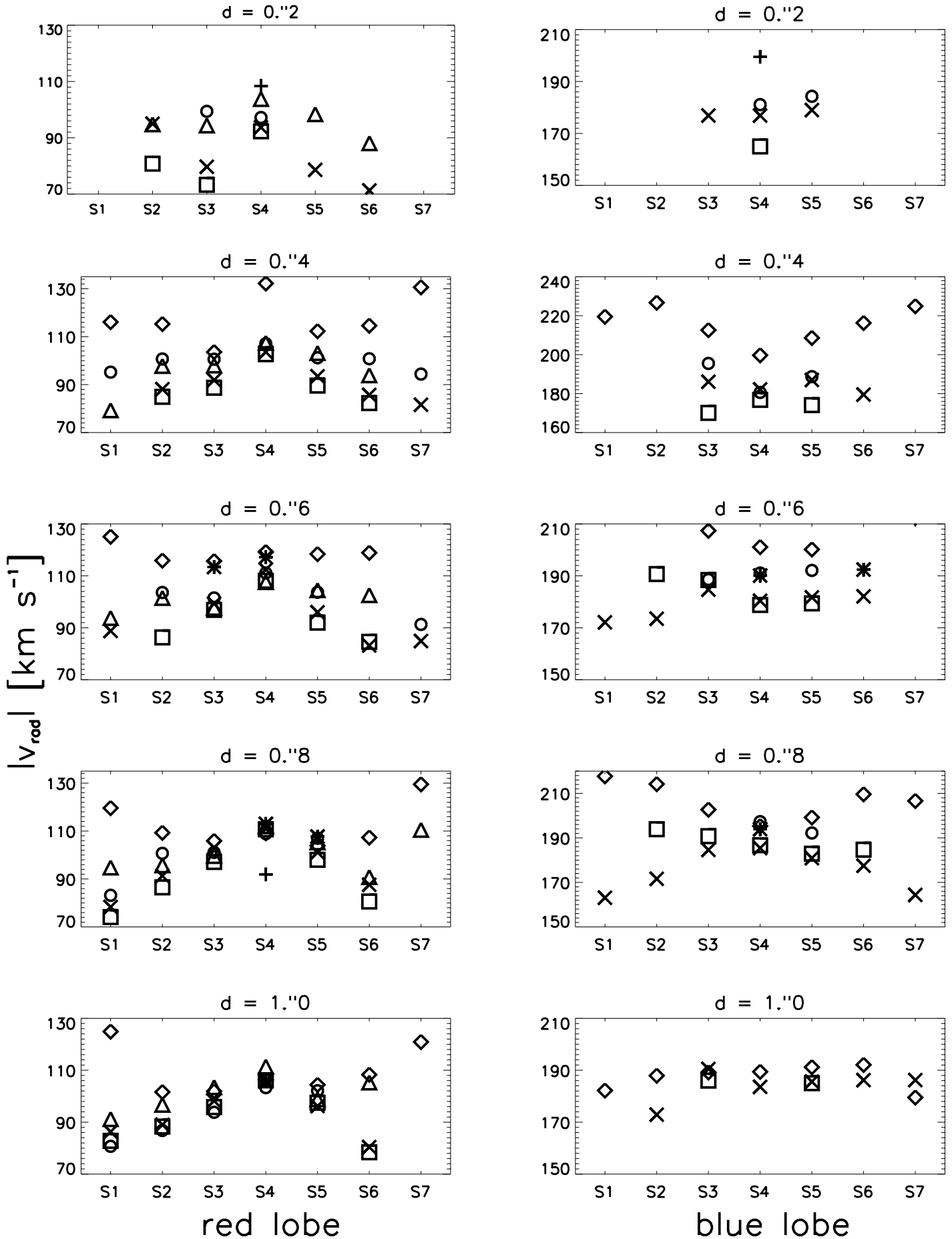

Fig. 3. Peak velocities for different slit positions and separations from the star. Crosses denote [SII] $\lambda 6731$, squares [SII] $\lambda 6716$, circles $[\mathrm{OI}] \lambda 6363$, triangles [OI] $\lambda 6300$, plus signs [NII] $\lambda 6548$, asterisks [NII] $\lambda 6583$, and diamonds $\mathrm{H} \alpha$.

For the given position angle the mimicked rotation would, however, have the opposite direction in comparison to the observed motion. This means that the true rotation velocities are in fact somewhat higher than those suggested by Figs. 4 and 5, and given in Table 1, strengthening our result even further.

Finally, the observed velocity shifts could in principle reflect a situation where the poloidal velocity field is not symmetric to the jet axis, but without any rotation. This can happen in asymmetric bow shock wings, but is very unlikely to occur along a distance of $300 \mathrm{AU}$, which is much larger than the knots of the RW Aur small-scale jet (Paper I).

As mentioned above, the data show a notable scatter in Figs. 4 and 5, and there are several possible explanations for this. Clumpiness, spatially unresolved shocks and variations of the poloidal velocity may play a role in producing the observed scatter. Some scatter would be present, however, even if the flow was laminar with the poloidal velocity smoothly decreasing from the axis to the jet borders ("onion-like" 


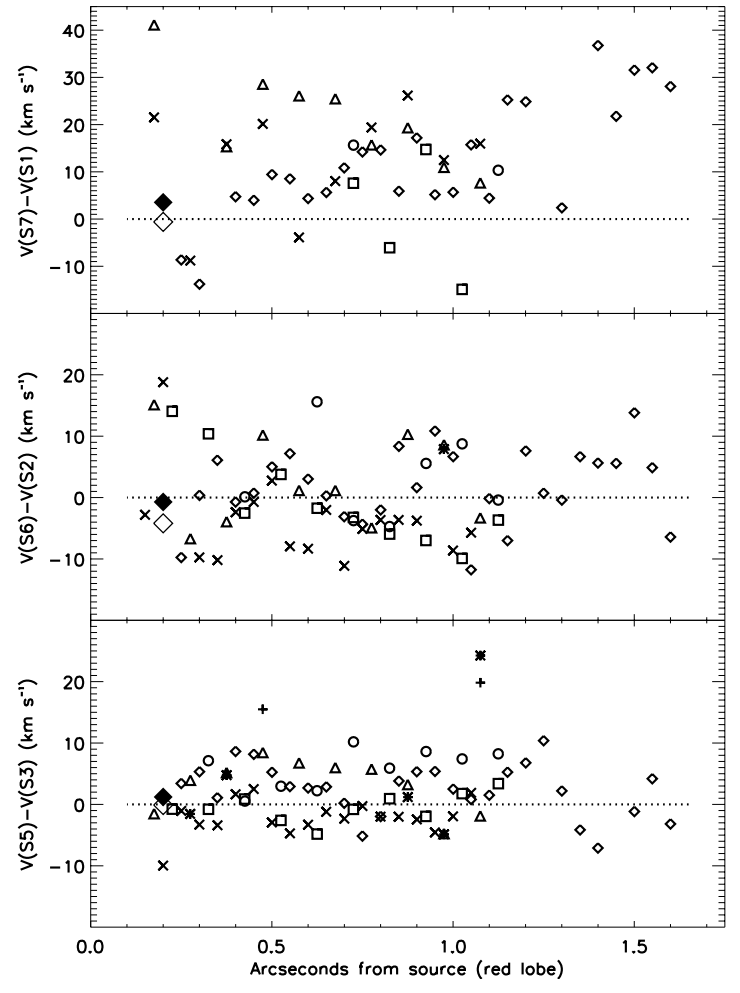

Fig. 4. Peak radial velocity differences in the redshifted outflow lobe as a function of separation from the source. Upper panel: difference between the values measured at slits $S 7$ and $S 1$, or at $30 \mathrm{AU}$ from the axis. Middle panel: $S 6-S 2$, corresponding to $20 \mathrm{AU}$ from the axis. Lower panel: $S 5-S 3$, or $10 \mathrm{AU}$ from the axis. The plot symbols have the same meaning as in Fig. 3. The large diamonds represent $\mathrm{H} \alpha$ at the stellar position, the open one before the correction for uneven slit illumination and the filled one afterwards.

kinematic structure), as predicted by the MHD stationary models. According to these models the layers located closer to the axis rotate faster, so different emission lines that trace different regions along the line of sight will also show different radial velocity shifts if measured at the same position on the sky. From Table 2 one can see that the rotation signatures are more pronounced in the $[\mathrm{OI}]$ and $[\mathrm{NII}]$ lines than in [SII] and $\mathrm{H} \alpha$. We have indeed found that the transverse $F W H M$ of the emission in $[\mathrm{OI}]$ and [NII] is smaller than the FWHM in [SII] (Fig. 4 of Paper I). The emission in $\mathrm{H} \alpha$ also presents a larger transverse extension. Thus at the same position across the jet, corresponding to two coupled slits, the " $[\mathrm{OI}]$ jet" and the "[NII] jet" are observed closer to their borders than the [SII] (and $\mathrm{H} \alpha$ ) jet. Pesenti et al. (2004) note that projection of the emission along a line of sight that crosses the beam of the jet tends to cancel out the radial velocity differences produced by rotation, this effect being more severe for distances closer to the axis and lower spatial resolution of the instrument. Therefore, at any point of observation, the rotation signatures will be more evident in [OI] and [NII], as the emission is less averaged across the line of sight in this case. The same projection effects can explain the fact that the evidence for rotation is weaker in the innermost slit pairs than it is in $S 7-S 1$. Note also that some values are negative in Table 2 for [SII] and $\mathrm{H} \alpha$. We interpret this as follows: we

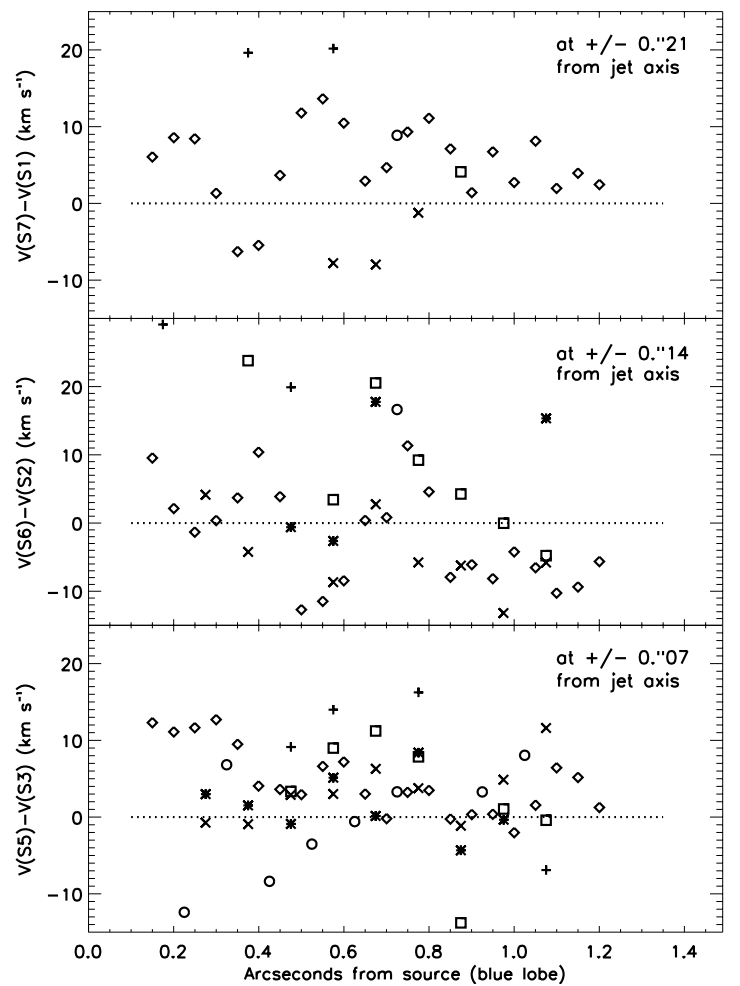

Fig. 5. Same as Fig. 4, but for the blueshifted outflow lobe.

Table 2. Velocity shifts (in $\mathrm{km} \mathrm{s}^{-1}$ ) in different emission lines, averaged over all separations from the star.

\begin{tabular}{llll}
\hline \hline Line & $S 7-S 1$ & $S 6-S 2$ & $S 5-S 3$ \\
\hline Redshifted lobe & & & \\
{$[\mathrm{OI}] \lambda 6300$} & 21.1 & 2.8 & 3.1 \\
{$[\mathrm{OI}] \lambda 6363$} & 13.0 & 3.0 & 5.9 \\
{$[\mathrm{NII}] \lambda 6548$} & & & 17.7 \\
$\mathrm{H} \alpha$ & 12.1 & 1.8 & 2.4 \\
{$[\mathrm{NII}] \lambda 6583$} & & 7.9 & 4.8 \\
{$[\mathrm{SII}] \lambda 6716$} & 0.3 & -0.6 & -0.5 \\
{$[\mathrm{SII}] \lambda 6731$} & 12.7 & -3.8 & -2.2 \\
Blueshifted lobe & & & \\
{$[\mathrm{OI}] \lambda 6363$} & 8.8 & 16.7 & 3.2 \\
{$[\mathrm{NII}] \lambda 6548$} & 19.9 & 24.5 & 8.1 \\
H $\alpha$ & 5.2 & -2.0 & 4.7 \\
{$[\mathrm{NII}] \lambda 6583$} & & 7.5 & 1.6 \\
{$[\mathrm{SII}] \lambda 6716$} & 4.1 & 8.1 & 2.6 \\
{$[\mathrm{SII}] \lambda 6731$} & -5.6 & -6.4 & 3.3 \\
\hline
\end{tabular}

have seen above that the set of slits is subject to an unwanted inclination of about 2 degrees with respect to the jet axis, in such a way that the observation of a non-rotating jet would produce a "false" negative shift. On the other hand, we have pointed out above that for any given slit pair the "[OI] jet" will show larger rotation shifts than the "[SII] jet", because of projection effects combined with a different spatial distribution of the emission. As explained above, in [OI] the rotation signature dominates over this inclination effect. In [SII] lines, instead, the rotation signature is very weak at the same position. This is because the aforementioned enhanced projection effects for the [SII] lines 
overwhelm the rotation here, and the net effect is the observation of a negative shift in [SII]. $\mathrm{H} \alpha$ may also be affected in the innermost slits. We have not attempted to correct for this effect, however, since the value of the "false" rotation depends on the kinematic model assumed for the jet. Pesenti et al. (2004) also predict rotational velocity shifts for "cold" and "warm" disk wind solutions. Our observed rotation velocities are intermediate between these two predictions, but closer to the warm disk wind solution. This point will be discussed further in Sect. 4.

Finally, we note that a decrease of the velocity shift with distance from the star is marginally seen in some of the lines and slit separations, as, for example, in the redshifted lobe for [OI] $\lambda 6300$ in the pair $S 7-S 1$ and $S 6-S 2$, and for [SII] $\lambda 6716$ in the $S 6-S 2$ pair, while for the blueshifted lobe a decrease is apparent in the $\mathrm{H} \alpha$ points for the $S 7-S 1$ pair and for [SII] $\lambda 6716$ in $S 6-S 2$. We note that such a behaviour is predicted by all the models of magneto-centrifugal acceleration as a consequence of the conservation of kinetic angular momentum at large distance from the Alfvèn surface (see below) and the widening of the flow. Given the scatter of our datapoints and their expected accuracy, however, we refrain from presenting a quantitative analysis of this aspect. Instead, in the rest of the paper we will use the radial velocity shifts measured in the first 0 . $^{\prime} 6-0.8$ from the star to derive as much information as possible about the physical properties of the launching region of the jet.

\section{Properties of the accretion/ejection structure}

From our results one can derive quantities useful to constrain the properties of the jet acceleration region. In particular it can be checked if the values obtained are consistent with the selfsimilar disk-wind models described, e.g. in Königl \& Pudritz (2000) and Ferreira (2002).

The launching region of the jet is sketched in Fig. 6. This figure illustrates the configuration of the nested magnetic surfaces attached to the inner portion of the accretion disk. The star is at the origin of a cylindrical coordinate system $(r, z, \phi)$, and the flow is assumed to be steady-state, axisymmetric and to satisfy the ideal MHD equations. The poloidal component of the magnetic field $\boldsymbol{B}_{\mathrm{p}}=B_{r} \boldsymbol{e}_{\boldsymbol{r}}+B_{z} \boldsymbol{e}_{z}$ is described by $\boldsymbol{B}_{\mathrm{p}}=$ $\nabla a \times \boldsymbol{e}_{\phi} / r$, where $a(r, z)=$ Const labels the magnetic surfaces, i.e. the surfaces enclosing a constant magnetic flux. The poloidal velocity and magnetic field are easily shown to be parallel, and they are related by the expression: $\rho \boldsymbol{v}_{\mathrm{p}}=k(a) \boldsymbol{B}_{\mathrm{p}}$, with $k$ constant along each magnetic surface. So the magnetic and flow surfaces are coincident and can also be labelled with their "footpoint" radius $r=r_{0}$, or the distance from the star on the disk where the surface is anchored. The mass in the visible jet is ejected from a region of the disk ranging from an inner radius $r_{0, \text { in }}$ to some outer radius $r_{0, \text { ext }}$ to be determined. The relevant mass fluxes are denoted by large grey arrows in the figure.

The disk and the rigidly anchored field lines rotate rapidly, and the centrifugal force makes the fluid parcels lifted from the disk surface (by the thermal pressure) be flung out along the open field lines. The matter flowing in the jet trails behind the field on a given surface. This generates a toroidal field component $B_{\phi}$ and, as a consequence, a "magnetic torque" that

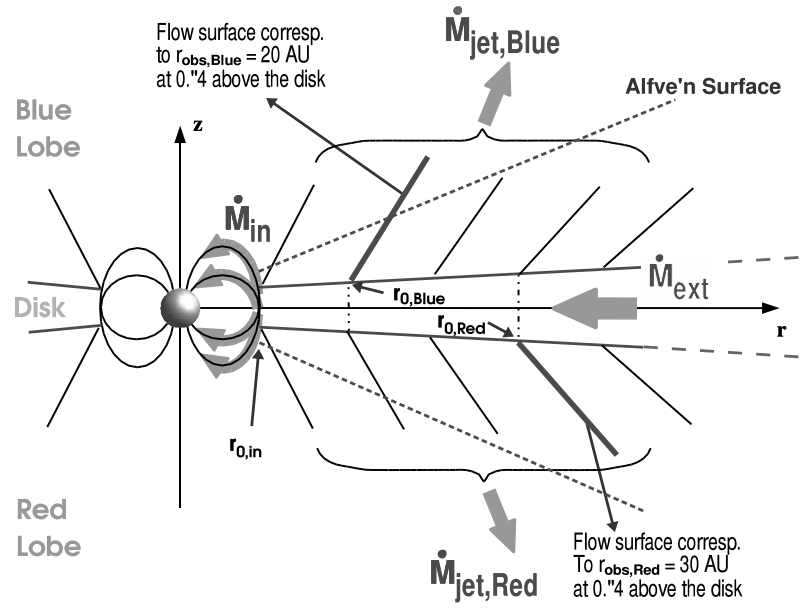

Fig. 6. Sketch (not to scale) of the configuration of the flow/magnetic surfaces (solid thin lines) attached to the inner section of the accretion disk around RW Aur A, according to the disk-wind scenario. Thick grey arrows indicate the mass flows. Note the different distances of the footpoints for the blue and red flows seen at $0 !^{\prime} 4$ above the disk. This is due to the asymmetry in the bipolar jet.

brakes the disk, and extracts energy and angular momentum from it. In the acceleration process, the matter reaches a point where the magnitude of the poloidal velocity equals the Alfvén poloidal velocity, or $\left|v_{\mathrm{p}}\right|=c_{\mathrm{A}}=\left|B_{\mathrm{p}}\right| / \sqrt{4 \pi \rho}$ ( $\rho$ is the mass density). The loci of such points constitute the so-called Alfvén surface. In the self-similar disk wind models (see, e.g., Casse $\&$ Ferreira 2000), this surface is conical, and its section is also indicated in Fig. 6 with a dashed line. Above the Alfvén surface, the inertia of the matter overcomes the magnetic forces, and the intensity of the toroidal field is increased substantially. This in turn generates a magnetic force ("hoop stress") directed toward the axis, that can collimate the flow.

The Alfvén surface lies at a few AU above the disk, a region that is still not resolved by observations. The datapoints we obtain in the first 0 !'2-0!'8 from the star, however, correspond to the region immediately above, and can be used to investigate the properties of the gas that has just been accelerated and collimated. The steps of the adopted procedure are illustrated in what follows. Measured and derived quantities are summarized in Tables 3 and 4, respectively.

\subsection{Footpoint radii}

The inner edge of the ejection region in the disk is commonly identified with the point where the disk is truncated by the interaction with the stellar magnetosphere. The corresponding disk annulus rotates at the same rate as the central object, and we will take this "corotation radius" $r_{\mathrm{c}}$ as a fiducial value for $r_{0, \text { in }}$. For a typical T Tauri star $r_{\mathrm{c}} \sim 0.03 \mathrm{AU}$ (Shu et al. 2000).

An estimate of the outer radius $r_{0, \mathrm{ext}}$ of the ejection region for the matter seen in optical lines can be derived from the combination of the poloidal and toroidal velocities $\left(v_{\mathrm{p}}, v_{\phi}\right)$ we measured in the external layer of the flow located at $r_{\text {obs }}$ from the jet axis. In a magneto-centrifugal wind the footpoint radius of the jet component located at $r_{\mathrm{obs}}$ from the axis with measured $v_{\phi}$ 
Table 3. Measured physical quantities at the jet base.

\begin{tabular}{lcccccc}
\hline \hline Jet lobe & $\begin{array}{c}r_{\mathrm{obs}} \\
(\mathrm{AU})\end{array}$ & $\begin{array}{c}v_{\phi}^{\mathrm{a}, \mathrm{b}} \\
\left(\mathrm{km} \mathrm{s}^{-1}\right)\end{array}$ & $\begin{array}{c}v_{\mathrm{p}}^{\mathrm{b}} \\
\left(\mathrm{km} \mathrm{s}^{-1}\right)\end{array}$ & $\begin{array}{c}M_{\star}^{\mathrm{c}} \\
\left(M_{\odot}\right)\end{array}$ & $\begin{array}{c}\dot{M}_{\mathrm{in}}^{\mathrm{d}} \\
\left(10^{-6} M_{\odot} \mathrm{yr}^{-1}\right)\end{array}$ & $\begin{array}{c}\dot{M}_{\text {jet }}^{\mathrm{e}} \\
\left(10^{-6} M_{\odot} \mathrm{yr}^{-1}\right)\end{array}$ \\
\hline Redshifted & $30 \pm 3$ & $16.8 \pm 5.3$ & $-130 \pm 10$ & & & $0.06 \pm 0.01$ \\
& & & & $1.3 \pm 0.1$ & $1.6 \pm 0.1$ & \\
Blueshifted & $20 \pm 3$ & $14.9 \pm 4.5$ & $260 \pm 10$ & & & $0.11 \pm 0.01$ \\
\hline
\end{tabular}

a Average of the significant values obtained from the FELs between $0 \prime^{\prime} 2$ and $0{ }^{\prime} 6\left(00^{\prime} 8\right)$ for the red (blue) lobe.

b The vector components are in a cylindrical coordinate system centred on the star and with positive $z$ axis oriented along the blue lobe (South-East in Fig. 1).

c From Woitas et al. (2001).

d From Hartigan et al. (1995).

e From Paper I.

Table 4. Derived physical quantities.

\begin{tabular}{|c|c|c|c|c|c|c|c|}
\hline Jet lobe & $\begin{array}{c}r_{0}^{\mathrm{a}} \\
(\mathrm{AU})\end{array}$ & $\begin{array}{c}v_{K}^{\mathrm{b}} \\
\left(\mathrm{km} \mathrm{s}^{-1}\right)\end{array}$ & $\begin{array}{c}\left|\dot{L}_{\text {jet,min }}\right|^{\mathrm{c}} \\
\left(M_{\odot} \mathrm{yr}^{-1} \mathrm{AU} \mathrm{km} \mathrm{s}{ }^{-1}\right)\end{array}$ & $\begin{array}{c}\dot{L}_{\text {disk,max }}^{\mathrm{d}} \\
\left(M_{\odot} \mathrm{yr}^{-1} \mathrm{AU} \mathrm{km} \mathrm{s}{ }^{-1}\right)\end{array}$ & $\xi^{\mathrm{e}}$ & $\lambda^{\mathrm{e}}$ & $B_{\phi} / B_{\mathrm{p}}^{\mathrm{f}}$ \\
\hline \multirow[t]{2}{*}{ Red } & $1.58 \pm 0.36$ & $27.0 \pm 3.2$ & $2.4 \pm 0.8 \times 10^{-5}$ & & $0.025 \leq \xi \leq 0.046$ & $12 \leq \lambda \leq 21$ & $3.8 \pm 1.1$ \\
\hline & & & & $6.6 \pm 1.5 \times 10^{-5}$ & & & \\
\hline Blue & $0.44 \pm 0.10$ & $51.2 \pm 6.2$ & $2.0 \pm 0.9 \times 10^{-5}$ & & $0.037 \leq \xi \leq 0.041$ & $13 \leq \lambda \leq 15$ & $-8.9 \pm 2.7$ \\
\hline
\end{tabular}

a Derived with the method in Anderson et al. (2003).

b Keplerian velocity at the footpoints $r_{0}$.

c Imposing that $\left|B_{\phi}\right|=0$ at the location of the observations and adopting the scalings in Ferreira \& Pelletier (1993) (see text).

${ }^{d}$ Considering only the portion of the disk from where the outflow seen at optical wavelengths originates.

e Assuming dominant magnetic torque; accuracy 20-22\%.

f At about 80 AU above the disk and 30 (20) AU from the axis for the red (blue) lobe. Coordinate system as in Table 3.

and $v_{\mathrm{p}}$ can be estimated with the relationship valid at large distances from the source provided by Anderson et al. (2003):

$$
\begin{aligned}
r_{0} \approx & 0.7 \mathrm{AU}\left(\frac{r_{\mathrm{obs}}}{10 \mathrm{AU}}\right)^{2 / 3}\left(\frac{v_{\phi}}{10 \mathrm{~km} \mathrm{~s}^{-1}}\right)^{2 / 3} \\
& \times\left(\frac{v_{\mathrm{p}}}{100 \mathrm{~km} \mathrm{~s}^{-1}}\right)^{-4 / 3}\left(\frac{M_{\star}}{1 M_{\odot}}\right)^{1 / 3},
\end{aligned}
$$

in which we will take $M_{\star}=1.3 \pm 0.1 M_{\odot}$ for the mass of RW Aur A (Woitas et al. 2001). This simple equation is valid under the condition $v_{\phi} \ll v_{\mathrm{p}}$, which in the context of disk winds is achieved as soon as $r>r_{\mathrm{A}}$, where the "Alfvén radius" $r_{\mathrm{A}}$ is the distance from the axis at which the magnetic/flow surface rooted at $r_{0}$ intersects the Alfvén surface. We note that at the sampled distances above the disk (50-100 AU), this asymptotic regime may not have yet been entirely reached by the outer streamlines of the flow. It can be shown, however, that in the case of "light" TTS winds, like the one treated here, the results given by the simplified formula above do not differ by more than a few percent from those obtained by a rigorous treatment of the conservation equations (see Anderson et al. 2003).

According to the expression above, in the red lobe, and for $r_{\mathrm{obs}}=30 \mathrm{AU}($ slits $S 7-S 1)$, one has $v_{p, \text { Red }} \sim 130 \pm 10 \mathrm{~km} \mathrm{~s}^{-1}$ (Paper I). For the toroidal velocity in the first 0.2-0.' 8 from the source we take $v_{\phi, \text { Red }}=16.8 \pm 5.3 \mathrm{~km} \mathrm{~s}^{-1}$, from the average of the significant datapoints corresponding to the forbidden lines ( $\mathrm{H} \alpha$ points are excluded because of the possible contamination with light coming from the star and accretion processes, and larger projection effects). From these values one obtains $r_{0, \text { Red }}=1.58 \pm 0.36 \mathrm{AU}$.

In the blue lobe the forbidden lines are quite faint in the $S 1$ and $S 7$ slits, but the needed velocity shifts can be extracted from the slit pair $S 6-S 2$. Taking the average of the significant values measured within 0 '. $^{\prime} 8$ from the star one obtains $v_{\phi, \text { Blue }}=14.9 \pm 4.5 \mathrm{~km} \mathrm{~s}^{-1}$ at $20 \mathrm{AU}$ from the axis. With $v_{p \text {, Blue }} \sim 260 \pm 10 \mathrm{~km} \mathrm{~s}^{-1}$, the origin of this portion of the flow appears to be located at $r_{0, \mathrm{Blue}}=0.44 \pm 0.1 \mathrm{AU}$. The values for both the red and blue lobes are in good agreement with analogous determinations by Coffey et al. (2004). We note that according to Pesenti et al. (2004) the toroidal velocity derived from measurements of the full velocity profile (as in our case) at $r_{\mathrm{obs}}=20 \mathrm{AU}$ from the axis may be underestimated by a maximum of $15 \%$ because of projection effects. A $15 \%$ correction to the toroidal velocity for the blue lobe, however, is smaller than our accuracy and will not be considered in the following.

It should also be remembered that the above calculation strictly gives only the footpoint of the flow surface for which the rotational velocity could be measured, and not the outer radius of the whole ejection region. For example, Takami et al. (2004) report about the discovery of a cold and slow wind component emitting in $\mathrm{H}_{2}$ lines, that surrounds the base of the optical jet from DG Tau. Such a component is probably anchored at larger footpoint radii than the optical component. Thus, the 
derived footpoint values may be conservatively considered as lower limits to the true extent of the launching region.

\subsection{Jet/disk angular momentum balance}

In the magneto-centrifugal approach, the angular momentum balance in all space can be written in conservative form as (Casse \& Ferreira 2000):

$\nabla \cdot\left(\rho r v_{\phi} \boldsymbol{v}_{\mathrm{p}}-\frac{r B_{\phi}}{4 \pi} \boldsymbol{B}_{\mathrm{p}}-r \boldsymbol{T}_{\mathrm{v}}\right)=0$,

where $\boldsymbol{T}$ is the "viscous" stress tensor inside the disk (Shakura \& Sunyaev 1973), and use has been made of the relation $\nabla \cdot r \boldsymbol{T}_{\mathrm{v}}=r\left(\nabla \cdot \boldsymbol{T}_{\mathrm{v}}\right)$. Viscosity can transfer the excess angular momentum radially from the inner to the outer region of the disk, but it is very difficult to quantify its importance (see the discussion in Casse \& Ferreira 2000). Our rotation measurements, however, allow us in principle to give rough estimates of the various contributions as follows.

We integrate Eq. (2) over the volume delimited laterally by the magnetic surfaces passing through $r_{0, \text { in }}$ and $r_{0 \text {,ext }}$, and above and below the disk by two surfaces, $S_{\text {Red }}$ and $S_{\text {Blue }}$, parallel to the disk plane and located in the observed regions at $\sim \pm 0$ '.4 AU from the source. We are thus led to calculate four surface integrals. By definition $\boldsymbol{B}_{\mathrm{p}} \cdot \boldsymbol{n}=0$ along the magnetic surfaces in all space, and in the jet $\boldsymbol{T}_{\mathrm{v}}=0$ (ideal conditions), and $\boldsymbol{v}_{\mathrm{p}} \| \boldsymbol{B}_{\mathrm{p}}$. Thus the integral over the lateral surfaces reduces to the fluxes through the annuli at $r_{0, \text { in }}$ and $r_{0, \text { ext }}$. Since in the disk the poloidal velocity is equal to the accretion velocity, the sum of the kinetic component of the flux through the annuli under consideration is

$\dot{M}_{\text {in }} r_{0, \text { in }} v_{\mathrm{K}, \text { in }}-\dot{M}_{\text {ext }} r_{0, \text { ext }} v_{\mathrm{K}, \text { ext }}=-\dot{L}_{\text {disk }}$,

where $v_{\mathrm{K}}=\left(G M_{*} / r_{0}\right)^{1 / 2}$ is the Keplerian velocity, and $\dot{M}_{\text {in }}$, $\dot{M}_{\text {ext }}$ are the mass accretion rates in the disk through the magnetic surfaces located at $r_{0, \text { in }}, r_{0, \text { ext }}$ respectively (cf. Fig. 6). Thus $\dot{L}_{\mathrm{disk}}$ represents the angular momentum that has to be extracted from the disk zone under consideration per unit time to allow for mass accretion. The component of the integral over the same surfaces due to viscosity will be indicated by $\dot{L}_{T}$, while the angular momentum fluxes in the jet lobes, $\dot{L}_{\mathrm{jet}, \mathrm{R}}$ and $\dot{L}_{\text {jet, }}$ result from the calculation of the integrals over $S_{\text {Blue }}$ and $S_{\text {Red }}$. In summary, one reduces to:

$\dot{L}_{\text {disk }}=\dot{L}_{\mathrm{T}}+\dot{L}_{\text {jet,Red }}+\dot{L}_{\text {jet,Blue }}$.

Our rotation measures can be used to give estimates of the angular momentum loss rate in the disk, $\dot{L}_{\text {disk }}$, and of the angular momentum extracted by the jet per unit time, $\dot{L}_{\mathrm{jet}}$. A comparison then between these quantities can give us a rough idea of the role played by the outflow, and, in turn, by viscosity, in the angular momentum balance.

To calculate $\dot{L}_{\text {disk }}$ we note that mass conservation leads to

$\dot{M}_{\text {ext }}=\dot{M}_{\text {jet,Red }}+\dot{M}_{\text {jet,Blue }}+\dot{M}_{\text {in }}$.

Referring to Fig. 6, we assume that all the mass flowing through the annulus at $r_{0, \text { in }}$ is deposited onto the star. Thus a good estimate for the inner mass flux is $\dot{M}_{\text {in }}=1.6 \times 10^{-6} M_{\odot} \mathrm{yr}^{-1}$, that was derived by Hartigan et al. (1995) from measurements of the veiling of photospheric lines caused by the accretion shock. The mass flux at the jet base in the two lobes, $\dot{M}_{\text {jet,Red }}$ and $\dot{M}_{\text {jet,Blue }}$, has been estimated in Paper I to be 0.6 and $1.1 \times 10^{-7} M_{\odot} \mathrm{yr}^{-1}$, respectively. We thus obtain $\dot{M}_{\text {ext }}=1.77 \times$ $10^{-6} M_{\odot} \mathrm{yr}^{-1}$. Taking conservatively $r_{0, \mathrm{ext}}=1.58 \pm 0.36 \mathrm{AU}$, and $r_{0, \text { in }} \geq 0.03 \mathrm{AU}$, one has

$$
\dot{L}_{\text {disk }} \leq 6.6 \pm 1.5 \times 10^{-5} M_{\odot} \mathrm{yr}^{-1} \mathrm{AU} \mathrm{km} \mathrm{s}^{-1} \text {. }
$$

We will now attempt an estimate of $\dot{L}_{\text {jet,Red }}$ and $\dot{L}_{\text {jet,Blue }}$. Since in the jet $\rho \boldsymbol{v}_{\mathrm{p}}=k(a) \boldsymbol{B}_{\mathrm{p}}$, this term can be written, for each lobe, as:

$\dot{L}_{\mathrm{jet}}=\int_{S}\left(r v_{\phi}-\frac{r B_{\phi}}{4 \pi k}\right) \rho \boldsymbol{v}_{\mathrm{p}} \cdot \boldsymbol{n} \mathrm{d} S$.

The MHD wind theory prescribes that the quantity in parentheses, which represents the total (kinetic plus magnetic) specific angular momentum, is also constant along the magnetic surfaces:

$l_{\text {jet }}(a)=\left(r v_{\phi}-r B_{\phi} / 4 \pi k\right)=r_{\mathrm{A}}^{2} \Omega_{0}$

where $\Omega_{0}=v_{K} / r_{0}$ is the angular speed of the disk at the base of the considered surface (see Königl \& Pudritz 2000). Equation (7) can thus be transformed to

$\dot{L}_{\mathrm{jet}}=\int_{S}\left(\frac{r_{\mathrm{A}}}{r_{0}}\right)^{2} r_{0} v_{\mathrm{K}} \rho \boldsymbol{v}_{\mathrm{p}} \cdot \boldsymbol{n} \mathrm{d} S$

The quantity $\frac{r_{\mathrm{A}}}{r_{0}}$ represents the "magnetic lever arm" of the flow, and it varies between 2 and 5 for typical flow parameters (see, e.g., Königl \& Pudritz 2000; Anderson et al. 2003; Pesenti et al. 2004). Assuming then that the flow surfaces are well-behaved and regularly nested, an upper limit to the angular momentum flux transported by the jet can be estimated from the measured jet mass flux rates, the derived values of $r_{0}$ and $v_{\mathrm{K}}$ at the outer footpoints (reported in Tables 3 and 4), and assuming a value for the magnetic lever arm. For example with $\left(r_{\mathrm{A}} / r_{0}\right)^{2}=10$ one obtains $\left|\dot{L}_{\text {jet,Red }}\right| \leq 2.6 \pm 0.8 \times 10^{-5} M_{\odot} \mathrm{yr}^{-1} \mathrm{AU} \mathrm{km} \mathrm{s}{ }^{-1}$ and $\left|\dot{L}_{\text {jet,Blue }}\right| \leq 2.5 \pm 1.2 \times 10^{-5} M_{\odot} \mathrm{yr}^{-1} \mathrm{AU} \mathrm{km} \mathrm{s}^{-1}$. When summed, this amounts already to about $80 \%$ of the excess angular momentum, $\dot{L}_{\mathrm{disk}}$, to be extracted from the portion of the disk from which the observed flow originates.

An interesting lower limit to the angular momentum transported by the jet can instead be given for the self-similar disk wind model of Ferreira \& Pelletier (1993). Before deriving this we note that the 3-D geometry of the $\boldsymbol{B}$ and $\boldsymbol{v}$ vectors in the magneto-centrifugal scenario is such that if the system rotates anticlockwise looking down the $+z$ axis $\left(v_{\phi}, v_{\mathrm{K}}>0\right)$, then $B_{\phi} B_{\mathrm{p}} / \rho v_{\mathrm{p}}=B_{\phi} / k(a) \leq 0$ in all space (i.e., in both the $z>0$ and $z<0$ domains), while the opposite holds for clockwise rotation. Therefore, for every possible configuration of the system the kinetic and magnetic terms in the specific angular momentum have opposite signs, or they add up in absolute value. Thus, for each lobe, a lower limit to $\left|\dot{L}_{\text {jet }}\right|$ is set assuming that the magnetic contribution is negligible. The integral in Eq. (7) can be computed exactly for the model of Ferreira \& Pelletier (1993). For this model $v_{\phi}, v_{z} \propto r^{-1 / 2}$ and $\rho \propto r^{(\xi-3 / 2)}$, where $\xi$ is the 
ejection index of the jet (see next section). In this case Eq. (7) reads:

$$
\begin{aligned}
\left|\dot{L}_{\mathrm{jet}}\right| \geq & \int_{S} r v_{\phi} \rho \boldsymbol{v}_{\mathrm{p}} \cdot \boldsymbol{n} \mathrm{d} S \approx v_{\phi}\left(r_{\mathrm{obs}}\right) r_{\mathrm{obs}}^{1 / 2} \\
& \times \frac{\xi}{\xi+1 / 2} \frac{r_{\mathrm{obs}}^{(\xi+1 / 2)}-r_{\mathrm{in}}^{(\xi+1 / 2)}}{r_{\mathrm{obs}}^{\xi}-r_{\mathrm{in}}^{\xi}} \dot{M}_{\mathrm{jet}},
\end{aligned}
$$

where the integral is calculated between the radius of the axial inner hole of the jet $r_{\text {in }}$, that is about $5 \mathrm{AU}$ at 0.4 from the star (Cabrit et al. 1999) and the radius of the observed external layer, $r_{\mathrm{obs}}$, which is $30 \mathrm{AU}$ and $20 \mathrm{AU}$ for the red and blue lobes, respectively. With $\xi=0.040$, a value similar to the one that will be derived for this jet in the next section, we obtain $\left|\dot{L}_{\text {jet,Red }}\right| \geq 2.4 \pm 0.8 \times 10^{-5} M_{\odot} \mathrm{yr}^{-1} \mathrm{AU} \mathrm{km} \mathrm{s}{ }^{-1}$ and $\left|\dot{L}_{\text {jet,Blue }}\right| \geq 2.0 \pm 0.9 \times 10^{-5} M_{\odot} \mathrm{yr}^{-1} \mathrm{AU} \mathrm{km} \mathrm{s}^{-1}$. Again summing these quantities up the total amounts to about two thirds of the angular momentum $\dot{L}_{\text {disk }}$, that has to be extracted from the examined portion of the disk per unit time.

The rough estimates above indicate that the mechanism generating the jet may indeed be capable of braking the disk with high efficiency. In the rest of this discussion we will assume that in the system under study all of the excess angular momentum is carried away by the wind, or, equivalently, that in the disk the magnetic torque is much larger than the viscous torque.

\subsection{Magnetic lever arm and ejection index}

Following the disk-wind formulation by Ferreira (1997, 2002), in a jet/disk structure like the one considered, the mass accretion flux through the disk, $\dot{M}_{\text {acc }}$, varies with distance from the star as $\dot{M}_{\text {acc }}=$ Const. $\times r_{0}^{\xi}$. The exponent $\xi$, that measures the local ejection efficiency, is called "ejection index", and regulates many of the physical properties of the wind. Under the assumption of dominant magnetic torque (see above), $\xi$ is simply related to $r_{\mathrm{A}} / r_{0}$ by the expression: $\left(r_{\mathrm{A}} / r_{0}\right)^{2}=\lambda \sim 1+1 / 2 \xi$.

According to the disk-wind models $0.005<\xi<0.5$, where the upper limit applies to self-similar solutions with subAlfvenic heating (warm solutions, see Casse \& Ferreira 2000). It is interesting to find limiting values for this parameter in our case.

A lower limit for $\xi$ is set by the conservation of mass in the disk/jet system. The external mass flux can be expressed through $\xi$ and $\dot{M}_{\text {in }}$ as

$\dot{M}_{\text {ext }}=\dot{M}_{\text {in }}\left(r_{0, \text { ext }} / r_{0, \text { in }}\right)^{\xi}$.

Since $r_{0, \text { in }}$ cannot be lower than the corotation radius $r_{\mathrm{c}}$, a lower limit for $\xi$ is given by:

$\xi \geq \frac{\ln \left(1+\left(\dot{M}_{\text {jet,Red }}+\dot{M}_{\text {jet,Blue }}\right) / \dot{M}_{\text {in }}\right)}{\ln \left(r_{0, \text { ext }} / r_{\mathrm{c}}\right)}$.

Taking for $r_{0, \text { ext }}$ the value of $r_{0}$ derived for the red lobe (1.58 AU) one obtains $\xi_{\text {min }}=0.025 \pm 0.005$. If instead we take $r_{0, \text { ext }}=0.44 \mathrm{AU}$, that is the lateral footpoint value for the blue lobe, one obtains $\xi_{\min }=0.037 \pm 0.005$. The value of $\xi_{\text {min }}$ may further differ if one considers that the full extent of the accretion/ejection region may be larger, due to the possible presence of a surrounding cold flow not visible at optical wavelengths (such as a $\mathrm{H}_{2}$ wind, cf. Takami et al. 2004). This would imply not only a larger $r_{0 \text {,ext }}$, but also a larger $\dot{M}_{\text {jet }}$, as one would have to consider also the contribution of such flow to the mass outflow rate. Both these quantities, however, are still undetermined for the case under study.

An upper limit for $\xi$ follows from the conservation of angular momentum in the jet region. For dominant magnetic torque the constant of motion can be expressed through $\lambda$ or $\xi$ in terms of the Keplerian rotation velocity at the footpoint:

$$
\begin{aligned}
\left|l_{\text {jet }}(a)\right| & =\left|r\left(v_{\phi}-\frac{B_{\phi}}{4 \pi k}\right)\right|=\lambda v_{\mathrm{K}} r_{0} \\
& =\left(1+\frac{1}{2 \xi}\right)\left(G M_{*}\right)^{1 / 2} r_{0}^{1 / 2}
\end{aligned}
$$

Now, as described in the previous paragraph, the kinetic and magnetic terms in $l$ always have opposite signs, so the condition $\left|B_{\phi}\right| \geq 0$ imposes an upper limit for $\xi$. For the red lobe, using $r_{0}=1.58 \mathrm{AU}$, one finds $\xi_{\max , \text { Red }}=0.046 \pm 0.011$, while for the blue lobe, with $r_{0}=0.44 \mathrm{AU}$, one obtains $\xi_{\text {max,Blue }}=$ $0.041 \pm 0.010$.

In summary, for the red lobe of the RW Aur jet $0.025 \pm$ $0.005 \leq \xi \leq 0.046 \pm 0.011$, or $12 \pm 3 \leq \lambda \leq 21 \pm 4$. At the same time, in the blue lobe one finds $0.037 \pm 0.005 \leq \xi \leq$ $0.041 \pm 0.011$, or $13 \pm 3 \leq \lambda \leq 15 \pm 4$. The derived values for $\xi$ are thus in the lower part of the range admitted by the model. Nevertheless, "cold" wind ejection (enthalpy negligible with respect to the kinetic energy at the footpoint) requires $\xi \lesssim 0.01$ (Casse \& Ferreira 2000). Thus our results are consistent with the idea that some sort of heating is provided at the base of the jet (i.e. the wind is "warm"), for example by a hot disk corona. The fact that an extra-heating seems to be required at this location has also been noticed by Garcia et al. (2001a) from the analysis of terminal poloidal velocities and jet total densities, and by Pesenti et al. (2004) from the analysis of rotation signatures in the DG Tau jet. If the heating is produced by an active disk, however, a certain amount of viscous dissipation must be allowed. Alternatively, the heating at the jet footpoints might be due to ambipolar diffusion and/or X-ray irradiation. See Garcia et al. (2001b) and Shang et al. (2002) for a discussion of these processes respectively.

\subsection{Properties of the magnetic field}

A quantity that can be derived directly from our measurements is the ratio $B_{\phi} / B_{\mathrm{p}}$ between the toroidal and poloidal components of the magnetic field vector at the location of the observations. This quantity indicates how much the lines of force are wrapped on a given magnetic surface. In the magnetocentrifugal scenario, the collimation of the jet is thought to arise from the hoop stress generated by the increase of the toroidal component of the magnetic field after the gas has passed through the Alfvèn surface (see, e.g. Königl \& Pudritz 2000). It is thus interesting to obtain from the observations an indication of the magnetic field configuration in the region just above the collimation zone. 
The ratio $B_{\phi} / B_{\mathrm{p}}$ can be derived using a further conservation law of general disk-wind theory (see Königl \& Pudritz 2000; Anderson et al. 2003):

$\left(v_{\phi}-\frac{B_{\phi} v_{\mathrm{p}}}{B_{\mathrm{p}}}\right) / r=\Omega_{0}$,

where $\Omega_{0}$ is the disk angular velocity at the footpoint. Introducing the values observed for $v_{\phi}, v_{\mathrm{p}}$ at $r=r_{\mathrm{obs}}$ in the two lobes, and using the corresponding $r_{0}$ to calculate $\Omega_{0}$, we find $B_{\phi} / B_{\mathrm{p}}=3.8 \pm 1.1$ in the red lobe, at $30 \mathrm{AU}$ from the axis and about $80 \mathrm{AU}$ from the disk. In the blue lobe, one finds $B_{\phi} / B_{\mathrm{p}}=-8.9 \pm 2.7$ at $20 \mathrm{AU}$ from the axis and at about $100 \mathrm{AU}$ above the disk (the signs refer to a cylindrical coordinate system with the positive $z$ axis oriented along the blue lobe).

If the poloidal magnetic field is almost entirely projected along the $z$ direction, these ratios would correspond to an inclination angle of the magnetic line of force with respect to the disk plane of about 15 degrees for the red lobe, and only 6-7 degrees for the blue lobe. The dominance of the toroidal component of the field appears to confirm that the flow is magnetically collimated, as prescribed by the magneto-centrifugal models. Also, the well-known asymmetry between the lobes of this jet is reflected in the magnetic configuration, as the field turns out to be more tightly wrapped in the blue lobe.

\section{Conclusions}

In this paper we have described a new combined observational/theoretical study of the rotation properties of the bipolar jet from RW Aur.

We have found rotation signatures in a set of spectra taken with HST/STIS with multiple slits oriented parallel to the flow axis, using techniques similar to the ones adopted in our first rotation study (of the DG Tauri jet, Bacciotti et al. 2002). We analyse the first $300 \mathrm{AU}$ of the jet (1'.5 at the distance of RW Aur), applying an updated version of the correction routines for uneven slit illumination. We find that both lobes rotate in the same direction (i.e. with different helicities), with toroidal velocities in the range $5-30 \mathrm{~km} \mathrm{~s}^{-1}$ at 20 and $30 \mathrm{AU}$ from the symmetry axis in the blue and red lobes, respectively. The sense of rotation is anti-clockwise looking from the tip of the blue lobe (PA $130^{\circ}$ North to East) down to the star. These results are confirmed by other HST/STIS observations from our group (with the slit placed transverse to the jet axis) presented in Coffey et al. (2004). Rotation is more pronounced in the data from the outermost slit pair, as expected because of projection effects along the line of sight (Pesenti et al. 2004). Also, rotation signatures are more evident in [OI] and [NII] lines than in $\mathrm{H} \alpha$ and [SII] lines. We interpret this result as due to the fact that $[\mathrm{OI}]$ and $[\mathrm{NII}]$ emission traces regions closer to the jet axis than $\mathrm{H} \alpha$ and [SII], and hence at a given position of the slit the jet is observed closer to its border in [OI] and [NII], which reduces the confusion due to projection.

The observed rotation favours widely known magnetocentrifugal models for the jet generation. Following the formulation in Anderson et al. (2003), the derived toroidal and poloidal velocities imply that the flow surfaces of the redshifted lobe observed at $30 \mathrm{AU}$ from the axis are rooted in the disk at about 1.6 AU from the star. The blueshifted flow observed at $20 \mathrm{AU}$ from the axis arises instead from a region in the disk at about $0.4-0.5 \mathrm{AU}$ from the star.

Making use of general principles underlying the disk-wind models, we have also derived other parameters useful to constrain the properties of the RW Aur accretion/ejection structure. We have estimated upper limits for $\dot{L}_{\text {jet }}$, the angular momentum transported by the visible jet lobes, and a lower limit for the same quantity in the special case of the self-similar disk-wind model of Ferreira \& Pelletier (1993). We compare these values with the angular momentum, $\dot{L}_{\text {disk }}$, that the region of the disk from which the visible outflow originates has to lose per unit time in order to accrete at the observed rate. We conclude that the jet is capable of extracting a consistent fraction (two thirds or more) of the excess angular momentum present in the disk.

Assuming moreover that all the excess angular momentum is carried away by the jet, we have also estimated the magnetic lever arm (expressed by the ratio $r_{\mathrm{A}} / r_{0}$ between the Alfvén and footpoint radii) for the self-similar disk wind model of Ferreira \& Pelletier (1993). We found this quantity to be in the range 3.6-3.9 for the blueshited lobe, and in the range 3.5-4.6 in the redshifted lobe (accuracy 20-25\%). Alternatively, the value of the ejection index $\xi=\mathrm{d} \ln \left(\dot{M}_{\text {acc }}\right) / \mathrm{d} r$ varies from 0.025 to 0.046 in the red lobe, and from 0.037 to 0.041 for the blue lobe (with the same accuracy). We caution that our determination of the magnetic lever arm (or ejection index) may be affected by the poor knowledge of the global extension of the launching region. In fact a component of the flow too cold to be visible at optical wavelengths may surround the observed jet. Nevertheless, the values determined for the optical component are in the range predicted by MHD models, and they also suggest that some heating is provided externally at the base of the flow. The nature of such heating, however, remains to be identified.

Finally, we have used our rotation measurement to derive information about the spatial configuration of the magnetic field in the examined region. In particular, using well-known conservation laws of the MHD disk wind theory we have derived the ratio $B_{\phi} / B_{\mathrm{p}}$ between the toroidal and poloidal components of the magnetic field at the observed locations in both lobes of the bipolar jet. We obtained $B_{\phi} / B_{\mathrm{p}}=3.8 \pm 1.1$ for the red lobe at $30 \mathrm{AU}$ from the axis and about $80 \mathrm{AU}$ from the disk and $B_{\phi} / B_{\mathrm{p}}=-8.9 \pm 2.7$ for the blue lobe, at $20 \mathrm{AU}$ from the axis and $100 \mathrm{AU}$ from the disk (in cylindrical coordinates, with positive $z$ along the blue lobe). The toroidal component of the magnetic field appears thus to be dominant, as expected for a magnetically collimated flow. In addition, the field seems to be more tightly wrapped in the blue lobe, reflecting the wellknown (but unexplained) asymmetries between the two lobes of this jet.

In summary, our observations and subsequent analysis appear to confirm once more the magneto-centrifugal scenario for the launching of YSO jets. To prove conclusively that jets solve the "angular momentum" problem in star formation, however, will require further detailed studies of a larger number of jets, and possibly at higher spectral and spatial resolution. 
Acknowledgements. J.E. and J.W. acknowledge support by the Deutsches Zentrum für Luft- und Raumfahrt (grant number 50 OR 0009), and T.R. and D.C. funding from Enterprise Ireland. We would like to thank the referee, Catherine Dougados, for a helpful and constructive report that led to a significant improvement of this paper. We are grateful to Jonathan Ferreira, Ralph Pudritz and Antonella Natta for useful comments. T.R., J.E., D.C., and J.W. wish to thank the Arcetri Observatory for hospitality during various visits.

\section{References}

Anderson, J. M., Li, Z. Y., Krasnopolsky, R., \& Blandford, R. D. 2003, ApJ, 509, 107

Bacciotti, F., Mundt, R., Ray, T. P., Eislöffel, J., \& Solf, J. 2000, ApJ, 537, L49

Bacciotti, F., Ray, T. P., Mundt, R., Eislöffel, J., \& Solf, J. 2002, ApJ, 576,222

Camenzind, M. 1990, Rev. Mod. Astron., 3, 234

Cabrit, S., Ferreira, J., \& Raga, A. 1999, A\&A, 343, L61

Casse, F., \& Ferreira, J. 2000, A\&A, 353, 1115

Cerqueira, A. H., \& de Gouveia Dal Pino, E. M. 2004, A\&A, 426, L25

Coffey, D., Bacciotti, F., Woitas, J., Ray, T. P., \& Eislöffel, J. 2004, ApJ, 604, 758

Dougados, C., Cabrit, S., Lavalley, C., \& Ménard, F. 2000, A\&A, 357, L61

Eislöffel, J., Mundt, R., Ray, T. P., \& Rodríguez, L. F. 2000, Protostars and Planets IV, 815

Ferreira, J., \& Pelletier, G. 1993, A\&A, 276, 625
Ferreira, J. 1997, A\&A, 319, 340

Ferreira, J. 2002, in Star Formation and the Physics of Young Stars, EAS Publ. Series, ed. J. Bouvier, \& J. P. Zahn (EDP Sciences), 3, 229

Garcia, P. J. V., Cabrit, S., Ferreira, J., \& Binette, L. 2001a, A\&A, 377, 609

Garcia, P. J. V., Ferreira, J., Cabrit, S., Binette, L., et al. 2001b, A\&A, 377,589

Hartigan, P., Edwards, S., \& Ghandour, L. 1995, ApJ, 452, 736

Hirth, G. A., Mundt, R., \& Solf, J. 1997, A\&AS, 126, 437

Königl, A., \& Pudritz, R. E. 2000, Protostars and Planets IV, 759

Kwan, J., \& Tademaru, E. 1988, ApJ, 332, L41

Kwan, J., \& Tademaru, E. 1995, ApJ, 454, 382

López-Martín, L., Cabrit, S., \& Dougados, C. 2003, A\&A, 405, L1

Marconi, A., Axon, D. J., Capetti, A., et al. 2003, ApJ, 586, 868

Petrov, P. P., Gahm, G. F., Gameiro, J. F., et al. 2001, A\&A, 369, 993

Pesenti, N., Dougados, C., Cabrit, S., et al. 2004, A\&A, 416, L9

Shang, H., Glassgold, A. E., Shu, F. H., et al. 2002, ApJ, 564, 853

Shakura, N. I., \& Sunyaev, R. A. 1973, A\&A, 24, 337

Shu, F. H., Najita, J. R., Shang, H., \& Li, Z. Y. 2000, Protostars and Planets IV, 789

Takami, M., Chrysostomou, A., Ray, T. P., et al. 2004, A\&A, 416, 213

Testi, L., Bacciotti, F., Sargent, A. I., Ray, T. P., \& Eislöffel, J. 2002, A\&A, 394, L31

Woitas, J., Leinert, Ch., \& Köhler, R. 2001, A\&A, 376, 982

Woitas, J., Ray, T. P., Bacciotti, F., Davis, C. J., \& Eislöffel, J. 2002, ApJ, 580, 336 (Paper I) 